\title{
FRUCTOSE AND CARDIOMETABOLIC DISORDERS: THE CONTROVERSY WILL, AND MUST, CONTINUE
}

\author{
Nicolas Wiernsperger, ${ }^{\mathrm{I}, \mathrm{III}}$ Alain Geloen, ${ }^{\mathrm{I}}$ Jean-Robert Rapin ${ }^{\mathrm{II}, \mathrm{III}}$ \\ doi: $10.1590 / \mathbf{S 1 8 0 7 - 5 9 3 2 2 0 1 0 0 0 0 7 0 0 0 1 3}$
}

Wiernsperger N, Geloen A, Rapin JR. Fructose and cardiometabolic disorders: the controversy will, and must, continue. Clinics. 2010;65(7):729-38.

The present review updates the current knowledge on the question of whether high fructose consumption is harmful or not and details new findings which further pushes this old debate. Due to large differences in its metabolic handling when compared to glucose, fructose was indeed suggested to be beneficial for the diet of diabetic patients. However its growing industrial use as a sweetener, especially in soft drinks, has focused attention on its potential harmfulness, possibly leading to dyslipidemia, obesity, insulin resistance/metabolic syndrome and even diabetes. Many new data have been generated over the last years, confirming the lipogenic effect of fructose as well as risks of vascular dysfunction and hypertension. Fructose exerts various direct effects in the liver, affecting both hepatocytes and Kupffer cells and resulting in non-alcoholic steatotic hepatitis, a well known precursor of the metabolic syndrome. Hepatic metabolic abnormalities underlie indirect peripheral metabolic and vascular disturbances, for which uric acid is possibly the culprit.

Nevertheless major caveats exist (species, gender, source of fructose, study protocols) which are detailed in this review and presently prevent any firm conclusion. New studies taking into account these confounding factors should be undertaken in order to ascertain whether or not high fructose diet is harmful.

KEYWORDS: Fructose; Triglycerides; Metabolic syndrome; Uric acid; Hypertension; Diet.

\section{INTRODUCTION}

In recent years, there has been increasing interest in the role of dietary fructose $(\mathrm{F})$ as a possible health risk. For decades, there has been a debate as to whether $\mathrm{F}$, which has a lower glycemic index than glucose $(G)$ and does not induce insulin secretion, is a good dietary alternative for diabetic patients. This important question has never been answered. More recently, it has been suggested that the worldwide burden of obesity and type 2 diabetes (T2DM) in young adults might be linked to a parallel increase in the consumption of artificially sweetened food, particularly soft drinks. ${ }^{1}$ Frequently in the United States, beverages (sodas and fruit juices) are sweetened by $F$, which is added as high F corn syrup (HFCS). HFCS has suddenly attracted

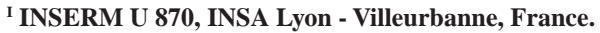

${ }^{\text {II }}$ Faculty of Pharmacy, University of Burgundy - Dijon, France.

II Association Remedes - Orlienas, France.

Email: nicolas.wiernsperger@free.fr

Tel: 33472310579

Received for publication on March 26, 2010

First review completed on April 06, 2010

Accepted for publication on April 06, 2010 attention regarding its responsibility in causing these metabolic disturbances. However, it must be noted here that this phenomenon particularly applies to the United States because countries in Europe and elsewhere have limited the use of HFCS. This is one reason, as will be seen later, for the ongoing discrepancies and as-yet uncertain conclusions.

The present review describes the various aspects of this important clinical question, exposes the reasons for some major discrepancies and details some reasons why we are missing firm conclusions and what must still be done to reach them.

\section{WHAT DO FRUCTOSE PHARMACODYNAMICS} TELL US?

\section{Intestines}

In normal alimentation, $\mathrm{F}$ is found in fruits and honey, either as pure $\mathrm{F}$ or as sucrose $(\mathrm{S})$, which is composed of $F$ and $G$ in equimolar amounts. Natural $F$ represents approximately $15 \%$ of total $\mathrm{F}$ intake. The higher sweetening capacity of $\mathrm{F}$ has led to its use as a frequent additive to liquid 
and solid foods. As will be seen later, this development has major consequences for answering the questions addressed in this review.

Although structurally similar, F behaves very differently from $\mathrm{G}$; it is absorbed in limited amounts, and most healthy subjects exhibit signs of intolerance at a daily $\mathrm{F}$ intake of approximately $50 \mathrm{~g},{ }^{2}$ free fatty acids and some amino acids favor the absorption of $\mathrm{F}^{3,4}$ which means that the threshold for intolerance may be even lower. $\mathrm{F}$ is transported via a specific glucose transporter, GLUT5, and exits enterocytes via GLUT2 transporters. It is important to note that chronic F intake upregulates GLUT5 expression. ${ }^{3}$ The lipidogenic effect of $F$ starts in the intestines because $F$ increases the levels of fatty acids associated with ApoB48, which are released as chylomicrons.

\section{Hepatocytes}

Once in the portal vein, $F$ is rapidly and almost completely extracted by the liver; the hepatic first pass is close to $100 \%$. Hepatocytes can transform $\mathrm{F}$ into various metabolites: glucose, glycogen, lactate, and fat. In contrast to $\mathrm{G}, \mathrm{F}$ is rapidly converted into triose-P independently of insulin. $\mathrm{F}$ is also a good precursor for gluconeogenesis because it generates lactate. Approximately $17 \%$ of $\mathrm{F}$ becomes glycogen, and approximately $20-50 \%$ of an F load leaves the liver as $\mathrm{G} .{ }^{5}$ In healthy individuals, compensatory mechanisms reduce other gluconeogenic pathways, maintaining constant total hepatic glucose production. Over a long period of time, however, $F$ decreases the insulin inhibition of hepatic glucose production, leading to hepatic insulin resistance. The phosphorylation of $\mathrm{F}$ requires high levels of ATP, leading to acute states of ATP depletion in hepatocytes and possible synthesis of uric acid (discussed below).

A typical feature of $\mathrm{F}$ is the induction of hypertriglyceridemia (HTG), notably because of reduced clearance. This is considered to be the most prominent negative effect of F. In addition to its effects on TGs, F also decreases HDL cholesterol levels.

\section{KUPFFER CELLS}

In the liver, the majority of cells are non-parenchymal cells. Sinusoids are lined with a dense net of Kupffer cells (KCs) and endothelial cells. KCs make up the largest proportion of macrophages in the body; they are strongly involved in detoxification and inflammatory reactions, liberating TNF, leukotrienes, interleukins and TGF- . ${ }^{6}$ Interestingly, $\mathrm{KCs}$ have a high density of insulin receptors and extract up to $50 \%$ of a $\mathrm{G}$ load. Thus, KCs may play an important but hitherto unrecognized and poorly understood role in the regulation of metabolic homeostasis. ${ }^{7}$ The few data sets that are presently available appear paradoxical. One might expect that $\mathrm{KC}$ impairment leads to metabolic disturbances because a blockage of KCs has been shown to result in hyperinsulinemia ${ }^{8}$. Furthermore, the elimination of KCs by clodronate-encapsulated liposomes was recently shown to provoke non-alcoholic steatotic hepatitis (NASH) due to the concomitant elimination of protective IL-10. ${ }^{9}$ Paradoxically, other models using gadolinium chloride to eliminate $\mathrm{KCs}$ have shown that this procedure improved insulin resistance and NASH induced by high fat ${ }^{10}$ or $\mathrm{S}$, one of the primary sources of F. ${ }^{11} \mathrm{TNF}$ is considered to be responsible for these disturbances. These data are intriguing, and it is difficult to know if this result is due to the use of gadolinium salt or to a massive overproduction of TNF, which would then overcome the beneficial effects of these cells. Whatever the explanation, we suggest that the role of $\mathrm{KCs}$ requires clarification because this very important hepatic tissue might represent a key element in the problem.

\section{WHAT DOES PATHOPHYSIOLOGY TELL US?}

\section{Metabolic disturbances}

High F (HF) is suspected to be involved in a series of diseases linked to metabolic disturbances, the best known being HTG and insulin resistance. As will be discussed later, several mechanisms are responsible for these direct and indirect effects of $\mathrm{F}$.

F metabolism does not seem to differ significantly between healthy and diabetic subjects. It impairs triglyceride (TG) clearance in both populations, but dyslipidemia is higher in subjects who are already resistant to insulin. ${ }^{12}$ Experimentally, HF diets have long been used to induce a metabolic state that is similar to human metabolic syndromes (MSs), namely insulin resistance and hyperlipidemia. However, this usually does not result in weight gain ${ }^{13}$ although the effects of $\mathrm{F}$ might vary among rat strains and some studies have reported weight gain in adolescents. ${ }^{14}$ In hamsters, fasting glycemia and weight were increased after chronic HF feeding. ${ }^{15}$ In mice, $\mathrm{F}$ increased adiposity and lipids in the liver, while $\mathrm{S}$ did not. ${ }^{16}$ This finding points to possible differences in species reactions to $\mathrm{HF}$, which is important to keep in mind when interpreting different sets of data. In rats, $\mathrm{F}$ is usually given in pellets, but it is also efficacious when added to drinking water. According to the concentration of $\mathrm{F}$ used, it is possible to generate groups of animals presenting various symptoms ranging from simple insulin resistance to full diabetes. When pure F was used instead of HFCS, no postprandial HTG was 
observed for oral intakes up to approximately $50 \mathrm{~g} / \mathrm{d} .{ }^{17} \mathrm{HTG}$ and fasting ApoB was, however, increased in overweight or obese women. ${ }^{18}$ Fasting increased levels of TG, VLDL-TAG and leptin without inducing insulin resistance or ectopic fat deposition in healthy males after four weeks of HF administration. ${ }^{19}$ More recently, however, the same group reported that just one week of HF administration increased ectopic fat deposition in the liver and skeletal muscle, with a greater effect seen in the offspring of diabetic parents than in healthy controls. ${ }^{20}$

The lipogenic activity of $\mathrm{F}$ can be demonstrated in vitro; adding HFCS-55 (a sweetener widely used in the United States) to hepatocytes leads to HTG and the elevation of lipogenic proteins and oxidative stress. At the same time, insulin resistance and endoplasmic reticulum stress are increased, and mitochondrial dysfunction is illustrated by cytochrome $\mathrm{C}$ release..$^{21}$ This finding fits with the mitochondrial disruption seen in human NASH.

In rats, the effects of $\mathrm{F}$ appear to be stronger in adults than in young animals. ${ }^{22} \mathrm{~F}$ is usually administered in high doses to rats to rapidly produce insulin resistance, but lower, more relevant levels of $\mathrm{F}$ also induce $\mathrm{G}$ intolerance if they are administered over long periods of time..$^{23} \mathrm{An} \mathrm{HF}$ diet in rats is considered to be a relevant model for human NASH, leading to typical hepatic lobular inflammation. ${ }^{24}$

In humans, a high intake of dietary $\mathrm{F}$ or sweetened fruit juice (but not whole fresh fruits) leads to impaired $G$ tolerance in genetically susceptible individuals..$^{25}$

In addition to dyslipidemia and insulin resistance/ metabolic syndromes, the $\mathrm{F}$ in a daily regimen of two or more colas has been shown to increase the risk of gallstones $^{26}$ and chronic kidney disease (stones). ${ }^{27}$

\section{Vascular disturbances}

Because of the high potential of $\mathrm{F}$ to glycate proteins compared to $\mathrm{G}$, concern has been raised about the possible roles of $\mathrm{F}$ in cardiovascular complications. The reason for this is partly because the acyclic (linear) form of the sugar (which is the glycating form) is larger in $\mathrm{F}$ than in $\mathrm{G}^{28,29} \mathrm{~A}$ meta-analysis showed that high $\mathrm{F}$ consumption increased cardiovascular risk by $24 \% .{ }^{30}$ The direct effects of $\mathrm{F}$ on protein glycation are questionable, however, because $\mathrm{F}$ is almost completely metabolized by the intestines and liver, leaving little if any intact $\mathrm{F}$ in the blood. HF is commonly assimilated into soft drinks; however, soft drinks (particularly colas) contain many other glycating (or glycated) agents. Thus, there is possible confusion about the real role of $\mathrm{F}$ in in vivo glycation. Some direct glycation might occasionally occur when food and drinks come into direct contact during eating. Indeed, the initial steps of the
Maillard reaction occur more rapidly with $\mathrm{F}$ than with $\mathrm{G}$, and the glycation of food proteins decreases their digestibility. ${ }^{31}$

The glycation of albumin occurs on Lys-524 and leads to the formation of carboxymethyllysine (CML). ${ }^{32}$ Interestingly, vegetarians show higher levels of CML than normal consumers, which can be explained by their high consumption of fruits, honey and vegetables. ${ }^{28} \mathrm{~F}$ is known to be involved in cataractogenesis. ${ }^{33}$ However, this is more likely to be due to intracellular $\mathrm{F}$ formation resulting from the sorbitol pathway; when F synthesis increases, it can glycate lens crystalline. ${ }^{34}$

Atheromatous plaques are increased in hypercholesterolemic rabbits when $\mathrm{F}$ is added to their diet. ${ }^{35} \mathrm{~F}$ is also suspected to cause hypertension (HT); acute F intake has been shown to increase blood pressure and heart rate in humans. ${ }^{36,37}$ In rats, an HF diet does not automatically generate HT; it seems that only some rat strains develop HT, which is dependent on an animal's age and diet protocol. This phenomenon may be due to the varying intra- and interspecies sensitivity toward F; indeed, rats, in contrast to humans, possess active uricase..$^{38,39}$

Vascular reactivity is modified with chronic HF diets, as endothelial dysfunction most likely occurs due to the inhibition of NO synthesis by F. ${ }^{40}$ Our experiments showed an abnormal reactivity of small terminal arterioles in the skeletal muscles of chronically F-fed rats. ${ }^{41}$

Finally, it is important to note that $\mathrm{F}$ also increases PAI$1,{ }^{42,43}$ a fibrinolytic factor that is known to be involved in the pathology of both metabolic and vascular disturbances of metabolic syndromes. ${ }^{44}$

\section{MECHANISMS INVOLVED IN FRUCTOSE-IN- DUCED DISTURBANCES}

\section{Direct effects in splanchnic organs}

In contrast to $\mathrm{G}, \mathrm{F}$ does not increase insulin secretion, which is considered to be potentially beneficial for diabetic patients. However, as described earlier, the primary negative side effect of $\mathrm{F}$ is its ability to increase lipid levels, especially TG. F increases both intestinal and hepatic production of atherogenic lipid particles ${ }^{45}$ in part through chylomicron assembly and secretion. ${ }^{46}$ Because F may even reduce insulin secretion, TG clearance also decreases. ${ }^{47}$ The quantitative effect of $\mathrm{F}$ on lipogenesis is a topic of debate. $F$ affects the partitioning of fatty acids toward their esterification, which has been shown in isolated perfused livers. ${ }^{48,49}$ In addition, $\mathrm{F}$ increases phosphofructokinase mRNA as well as G-6PDH and CHREB $^{50}$ and fatty acid synthase activities. ${ }^{51}$ These effects lead to hepatic lipid loading, resulting in NASH (of which an HF diet is a 
relevant model in rats). The combined negative effects on liver lipid content and lactate and lipid output ${ }^{52}$ makes this organ a crucial effector of HF-induced metabolic disturbances. Independently of an MS diagnosis, patients presenting NASH were found to consume more soft drinks than healthy subjects. ${ }^{53}$ Interestingly, when moderate intake levels of various sweeteners were compared in rats during a ten-week period, both $\mathrm{F}$ and HFCS increased alanine aminotransferase activity, although no other differences were observed. ${ }^{54}$ These data show that the first premises of fatty liver can be seen even following moderate $\mathrm{F}$ consumption. Figure 1 depicts the major fate of $\mathrm{F}$ in the splanchnic area as well as some differences between $\mathrm{F}$ and $\mathrm{G}$.

Recently, the involvement of intestinal bacterial flora in the regulation of metabolic homeostasis has been the subject of intense interest. ${ }^{55,56} \mathrm{In}$ particular, studies have revealed that NASH is associated with intestinal bacterial overgrowth and increased intestinal permeability, leading to the endotoxindependent activation of KCs. ${ }^{55}$ This effect was also shown with an HF diet in rats. ${ }^{57}$ Thus, intestinal effects may add to hepatic effects as causal effectors of HF-linked metabolic abnormalities.

While $\mathrm{F}$ intolerance does not seem to differ between normal subjects and those suffering from irritable bowel syndrome, ${ }^{58,59}$ an improvement in F intolerance was observed in $1 / 3$ of these patients after $F$ restriction. ${ }^{60}$ Conversely, $F$ and fructans may induce irritable bowel syndrome and intestinal permeability. ${ }^{55,61-63}$

\section{Indirect metabolic effects}

By increasing the liver lipid content and provoking HTG, $F$ leads to global metabolic derangements that are typical of MSs, eventually leading to T2DM. Several studies have shown that HF induces hepatic insulin resistance, resulting in an elevated $\mathrm{G}$ output due to the lack of gluconeogenesis suppression. Once in the circulation, TGs may accumulate in skeletal muscle cells and induce peripheral insulin resistance.

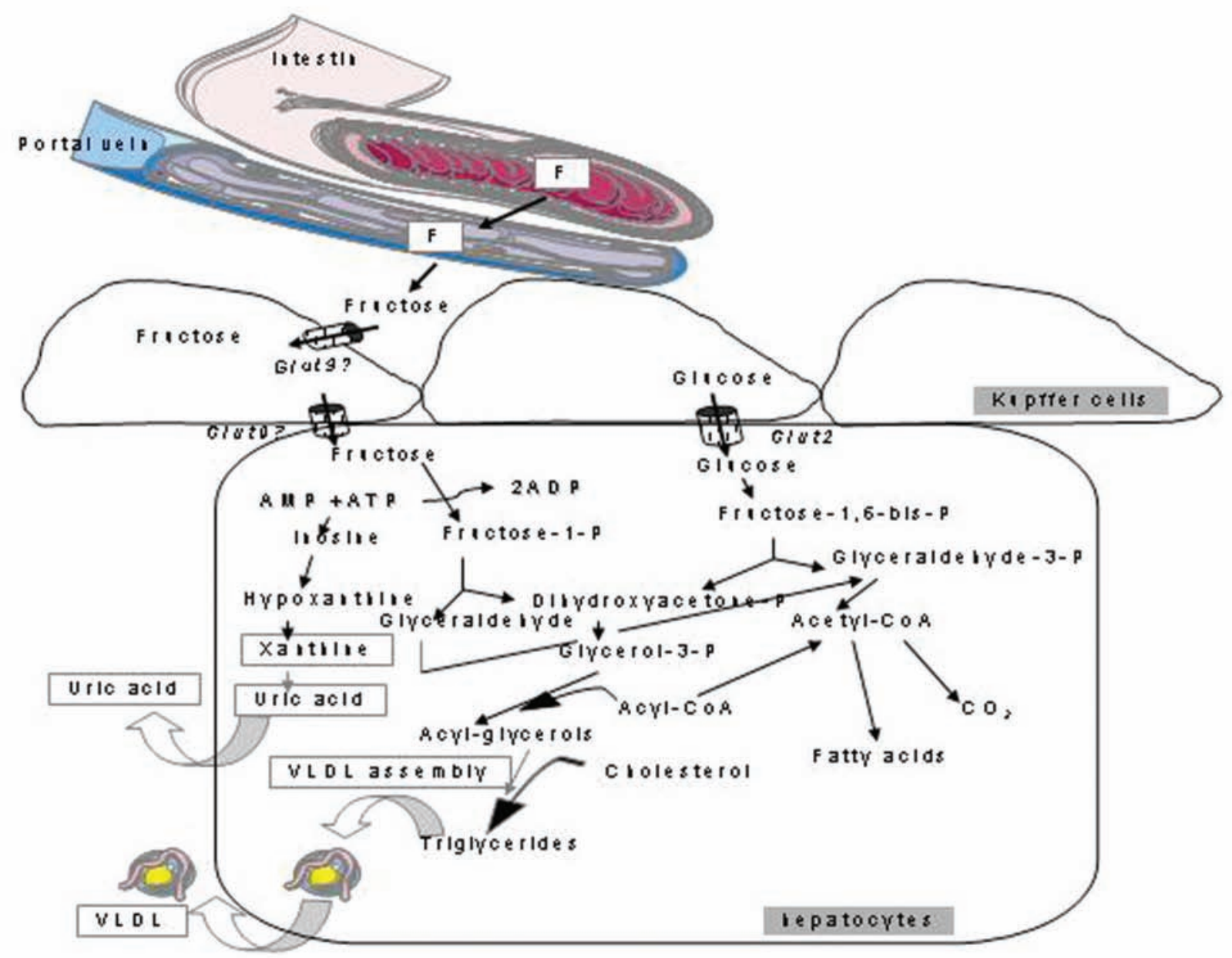

Figure 1 - Fructose is absorbed in the intestine by GLUT5 and exits enterocytes via GLUT2 transporters. It is taken up by Kupffer cells and hepatocytes, where it enters various metabolic pathways that largely differ from those of glucose. Fructose is partly transformed into uric acid, which may cause metabolic and vascular disturbances in peripheral tissues. 


\section{High energy intake}

It has been suggested that energy intake is higher with oral $\mathrm{F}$ than with oral $\mathrm{G}$. The reasons for this belief are the following: 1) the thermic effect of $F$ is higher than $G$ because of high ATP consumption, and 2) it was observed that the satiety phenomenon normally regulating food intake did not always operate after chronic F. F lowers ghrelin and leptin levels to a lesser degree than does $\mathrm{G}$, resulting in lower food intake suppression. ${ }^{1,3}$ This phenomenon applies mainly to liquid $\mathrm{F}$, leading to a higher energy balance than $\mathrm{F}$ in solid food. ${ }^{64,65}$ When sweetened beverages were taken together with meals, a higher mean energy intake of $104 \mathrm{kcal}$ was measured. ${ }^{66}$ Nevertheless, the "energy balance" hypothesis of HF is still a subject of debate. ${ }^{67}$

\section{Is uric acid the culprit?}

Uric acid (UA) is a major antioxidant, but, like most molecules of this category, ${ }^{68}$ it can also induce opposite effects if concentrations are sufficiently high. ${ }^{69}$ There are likely to be concentration thresholds delineating positive from negative effects of such substances; acute and even relatively high UA levels protect against oxidative stress ${ }^{70}$, but chronically elevated UA levels are linked to MSs, reduced adiponectin and elevated E-selectin, in parallel with positive effects such as reduced nitrotyrosine and increased total antioxidant capacity. ${ }^{71}$ Thus, high UA levels could be a compensatory mechanism that counteracts the oxidative stress related to metabolic or vascular disturbances. ${ }^{72}$

In vitro UA increases NADPH oxidase activity and oxidative stress in adipocytes, leading to increased p38 MAP kinase activity and insulin resistance. ${ }^{73}$ UA levels correlate with 24-h urinary excretion, waist circumference, insulin concentrations and the HOMA index, which are all indices of a MS. ${ }^{74}$ High insulin levels reduce renal UA excretion, suggesting that hyperuricemia results from a combination of stimulated formation and reduced elimination. In adolescents, body mass index and a number of MS components correlate with UA. ${ }^{75}$ In the San Bernardo Study, UA was a good predictor of T2DM in older adults with impaired fasting glycemia. ${ }^{76}$ Elevated UA was shown to be linked to NASH independently of body mass index. ${ }^{77-80}$ In elderly male subjects, high levels of UA were correlated with MS components and elevated levels of PAI- $1 .{ }^{81}$ Thus, it seems that at any age, high levels of UA are linked to MS. Decreasing UA levels in rats with allopurinol prevents MS, as shown by reductions in insulin, TG and weight. ${ }^{40}$

On the vascular side, high levels of UA are linked to cardiovascular diseases, especially HT. ${ }^{82-86}$ If patients are already at risk, then UA represents a serious problem. ${ }^{87,88}$
Mechanisms that are responsible for the deleterious $\mathrm{UA}$ effects are linked to increased $\mathrm{C}$ reactive protein, inflammation and reduced NO production, thereby hampering vascular reactivity. ${ }^{38,82,84,89}$

HF increases UA formation as a consequence of ATP consumption and high synthesis of AMP, which is then deaminated to form UA. ${ }^{3,90}$ Several studies have shown that HF increases UA levels. However, in the first phase of the Nanes III study, MS was not linked to UA in subjects who consumed diet soft drinks ${ }^{91}$. UA is known to be responsible for gout due to the deposition of monosaccharide urate crystals; this effect is strongly related to diet. ${ }^{92} \mathrm{In}$ a prospective cohort study over 12 years, $\mathrm{F}$ intake was correlated with gout regardless of whether sweetened soft drinks, fruit juices or F-rich fresh fruits were consumed. ${ }^{77}$ However, while this manuscript was in its final phase, a new publication showed that an analysis of more recent data from the Nanes III database (>9000 subjects) failed to confirm an association between $\mathrm{F}$ and UA. Thus, over a long time period, UA might not be as harmful in an HF diet as was initially claimed, which is in agreement with other reports. ${ }^{93}$

\section{FRUCTOSE VS. OTHER SUGARS}

A long-lasting controversy exists regarding the harmfulness of $\mathrm{F}$ versus other sugars because HFCS is now commonly used to sweeten drinks and food. Two questions must therefore be addressed: 1) how does "pure" F compare with $\mathrm{S}$, a sugar containing one moiety of $\mathrm{F}$, and 2) how does F compare with G? It is evident that such studies can easily be biased if energy equivalents are not matched in the clinical investigations. Most studies comparing $\mathrm{F}$ and $\mathrm{S}$ have shown that both sugars behave in essentially similar manners, pointing to the fact that $\mathrm{F}$ is actually the culprit. ${ }^{94-97}$ No differences in energy balance were found between HFCS, S and milk. ${ }^{98}$ When compared to G, HFCS and S always lead to higher TG or glucose/insulin responses over a 24-h period. ${ }^{99}$ In a study investigating the effects of $34 \%$ $\mathrm{F}$ in 3 different diets ( $\mathrm{F}, \mathrm{F}+\mathrm{G}$ and $\mathrm{S})$, a reduction in insulin sensitivity in rats was observed in every regimen. ${ }^{97}$ Several studies have compared F and $\mathrm{G}$, and in a one-week study, F increased VLDL more than G, but intramuscular lipids were higher with G. ${ }^{100}$ Essentially the same qualitative difference was found in another study that extended the comparison to one month. ${ }^{96}$ Giving sweetened beverages to overweight or obese subjects for up to 10 weeks showed that $\mathrm{F}$ but not $G$ increased lipid synthesis and visceral adiposity and reduced insulin sensitivity; $\mathrm{G}$ administration led to increased subcutaneous fat, which is relatively harmless. ${ }^{101}$ When $\mathrm{F}$ or $\mathrm{G}$ was administered with meals, obese patients showed lower insulin and leptin levels but increased postprandial 
TGs; HTG lasted for over 24 hours in the insulin-resistant subgroup. ${ }^{12}$

Altogether, these data show that $\mathrm{F}$ has roughly the same harmfulness whether it is administered as a concentrate such as HFCS or as sucrose. When the comparison is made with $\mathrm{G}$, however, there are strong qualitative differences.

\section{Therapeutic effects}

Various clinical studies have shown that reducing $\mathrm{F}$ intake improves ones' metabolic situation, which is expected. Thus, intestinal side effects due to $\mathrm{F}$ intolerance or excess $\mathrm{F}$ in food can be improved by a F-limited diet. Metabolic changes such as abdominal fat deposition, insulin resistance, oxidative stress and HT are improved by treatment with the antidiabetic metformin. ${ }^{37,102-105}$

\section{Caveats and limits of the actual dataset}

As is often found in nutritional studies, many factors, which can be controlled or not controlled, can bias interpretations of data. As a consequence, our present knowledge must consider and eventually investigate these factors before delivering clear-cut conclusions.

\section{Species}

As discussed above, differences in metabolic reactions to F have been described among various species: intra-species differences among rat strains, inter-species differences among mice, rats and hamsters and differences between rodents and humans. The presence of uricase in rats usually requires the utilization of high $\mathrm{F}$ concentrations in these animals, particularly when researchers wish to produce pathological individuals rapidly.

\section{Type of food}

It is known that the source of F partly determines its harmfulness; not only is it different among fresh fruits, fruit juices and sweetened soft drinks, but some sources, such as honey, appear to be much safer than others. Furthermore, several studies have shown that hormonal and metabolic reactions are more pronounced with liquid $F$ intake than with solid F intake, likely because of the bypass of the satiety process with liquid $\mathrm{F}$.

Another important caveat is that most clinical studies use soft drinks as the source of F. However, usually other parameters, such as daily alimentary habits or lifestyle (e.g., food type, activity levels) can affect ones' metabolism. Focusing on F soft drinks as the sole cause of metabolic modifications limits the power of the data. The same holds true for vascular disturbances because colas and other soft drinks contain many other molecules that could interfere with vascular reactivity or induce glycation.

\section{Protocols}

\section{a) Study Duration}

One major aspect of study protocols is the duration of the study. Available data have been obtained primarily from acute (2-24 h) or mid-term (1-4 weeks) administration of F; only some data have been obtained from longterm investigations. It is clear that acute and short-term administration of moderate and even high $\mathrm{F}$ will cause tissues to react against what they might sense as an aggression. We know that metabolic disturbances take years to manifest as clinical symptoms due to stepwise developing compensations, metabolic adaptations and new equilibrium levels (the development of T2DM is a good example). Concerning F, the UA aspect may also illustrate how an initially positive reaction (hyperuricemia) may turn into a negative one. Acute and chronic F administration can yield different results. This has been observed for TG levels, which were increased in acute but not chronic HF diets. ${ }^{101}$ Moreover, a recent report did not observe a correlation between $\mathrm{F}$ and UA over long time periods. ${ }^{93}$

\section{b) Gender/Populations}

The relatively large number of studies published in recent years can be divided into various categories of subjects or patients: young, old, healthy, overweight/obese, and diabetic. It is understandable that data might look very different among these populations. Ethnicity is another possible confounder because we know that certain populations have greater risks of developing insulin resistance, obesity and/ or MSs. For F, gender may play a role. Differences in most domains of physiology between males and females have become increasingly recognized in the last 5-10 years. This may also apply to HF. For example, it is known that female rats are largely protected compared to males. Insulin sensitivity decreases to a much larger extent in males ${ }^{106}$, and generally only males develop HT. The same effect holds true for humans; a careful examination of the literature shows a more severe picture in males than in females. In adolescents, weight gain under HF conditions was higher in males than in females. ${ }^{14}$ Furthermore, TG levels were higher in males than in females under HFCS or $\mathrm{S}$ administration conditions. ${ }^{99}$ The incidences of hyperuricemia and MS were higher in males than in females. ${ }^{79}$ Finally, in a study of Japanese subjects, UA increased the incidence of MS in males but not in females. ${ }^{107}$ 


\section{c) Dosage}

As discussed previously, $\mathrm{F}$ is only partly absorbed and rapidly leads to intestinal intolerance, which illustrates the fact that an HF condition is very different from a low F condition. However, most studies deal with high or very high $\mathrm{F}$ concentrations, which are not relevant to daily $F$ consumption in humans, even if one considers 'colaaddicted' young people. Moreover, many studies were performed in the United States using HFCS, a sweetener that is not used or is used only in limited amounts in many other countries. Thus, extreme caution should be taken when trying to develop firm conclusions ${ }^{17}$ In rats, moderate intake of various sweeteners (F, HFCS or agave) for 3 nights per week during a 10 -week period increased serum alanine aminotransferase levels, but no other serious disturbances, such as those seen with HF, were observed. ${ }^{54}$ In humans, the deleterious effects seen with $20-25 \% \mathrm{~F}$ as an energy source were not observed when more realistic quantities of $4-12 \%$ F were investigated. ${ }^{96}$

\section{CONCLUSIONS}

The controversy that has existed for the last 10 years regarding the potential harmfulness of excess fructose is legitimate in view of the dramatic increases in both sweetened beverage consumption and the burdens of obesity, MS and T2DM. ${ }^{108}$ There is no doubt from many preclinical and clinical studies that HF can induce negative effects on both energy metabolism and blood vessels (summarized in Figure 2).
Unfortunately, the vast majority of the studies were performed using protocols in which the duration or dosage of $\mathrm{F}$ administration was not relevant to common daily usage in the general population. Nevertheless, the merit of these studies was to provide valuable information as to what can be expected from excessive $\mathrm{F}$ intake and the nature of the underlying mechanisms. However, many important confounding factors were ignored, which makes data interpretation too hazardous to draw conclusions about the deleterious nature of HF.

Assuming there is no $\mathrm{F}$ intolerance, we infer from the available dataset that $\mathrm{F}$ is harmless in healthy individuals, at least at levels below 50-100 g/d. However, it also appears clear that individuals at risk for metabolic syndromes, T2DM or cardiovascular diseases should be cautious because evidence shows that they are much more susceptible to $\mathrm{HF}$ than the general population. Thus, the controversy continues, and new protocols that take into account our present knowledge and eliminate the aforementioned confounding factors are required. From the available literature, four main aspects of $\mathrm{F}$ intake appear to be particularly important: a) the use of "natural" F sources (sucrose) instead of HFCS or sweetened soft drinks (which contain many additional substances), b) the use of relevant $\mathrm{F}$ concentrations based on existing data about common daily $\mathrm{F}$ consumption in the general population, c) the mixing of male and female subjects and $\mathrm{d}$ ) the investigation of moderately elevated $\mathrm{F}$ over long periods of time.

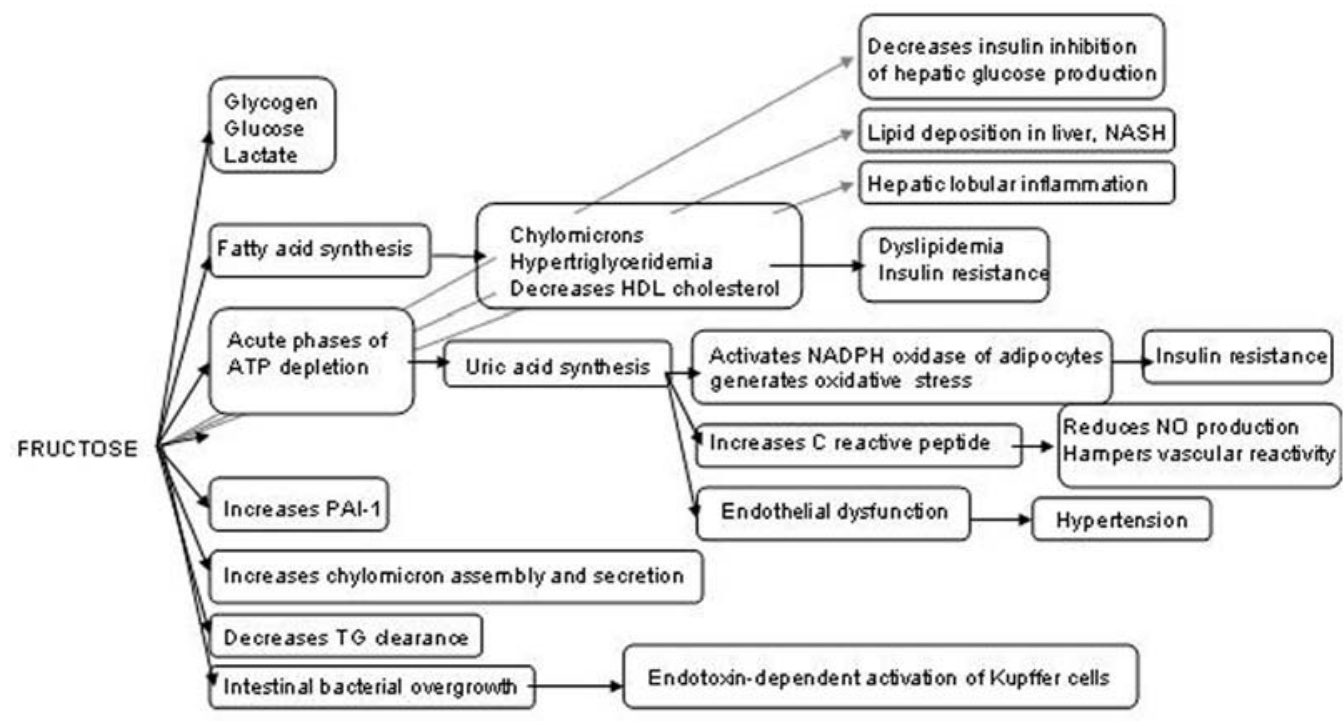

Figure 2 - Summary of short- and long-term metabolic and vascular disturbances and the underlying mechanistic pathways induced by high fructose intake. 


\section{REFERENCES}

1. Tappy L, Le KA. Metabolic effects of fructose and the worldwide increase in obesity. Physiol Rev. 2010;90:23-46.

2. Rao SS, Attaluri A, Anderson L, Stumbo P. Ability of the normal human small intestine to absorb fructose: evaluation by breath testing. Clin Gastroenterol Hepatol. 2007;5:959-63.

3. Johnson RJ, Perez-Pozo SE, Sautin YY, Manitius J, Sanchez-Lozada LG, Feig DI, et al. Hypothesis: could excessive fructose intake and uric acid cause type 2 diabetes? Endocr Rev. 2009;30:96-116.

4. Skoog SM, Bharucha AE. Dietary fructose and gastrointestinal symptoms: a review. Am J Gastroenterol. 2004;99:2046-50.

5. Nuttall FQ, Khan MA, Gannon MC. Peripheral glucose appearance rate following fructose ingestion in normal subjects. Metabolism. 2000;49:1565-71.

6. Kmiec Z. Cooperation of liver cells in health and disease. Adv Anat Embryol Cell Biol. 2001;161:III-XIII, 1-151.

7. Spolarics Z, Ottlakan A, Lang CH, Spitzer JJ. Kupffer cells play a major role in insulin-mediated hepatic glucose uptake in vivo. Biochem Biophys Res Commun. 1992;186:455-60.

8. Cornell RP. Mechanisms of acute hyperinsulinemia after Kupffer cell phagocytosis. Am J Physiol. 1980;238:E276-83

9. Clementi AH, Gaudy AM, van Rooijen N, Pierce RH, Mooney RA. Loss of Kupffer cells in diet-induced obesity is associated with increased hepatic steatosis, STAT3 signaling, and further decreases in insulin signaling. Biochim Biophys Acta. 2009;1792:1062-72.

10. Neyrinck AM, Cani PD, Dewulf EM, De Backer F, Bindels LB, Delzenne NM. Critical role of Kupffer cells in the management of diet-induced diabetes and obesity. Biochem Biophys Res Commun. 2009;385:351-6.

11. Huang W, Metlakunta A, Dedousis N, Zhang P, Sipula I, Dube JJ, et al. Depletion of liver Kupffer cells prevents the development of diet-induced hepatic steatosis and insulin resistance. Diabetes. 2010;59:347-57.

12. Teff KL, Grudziak J, Townsend RR, Dunn TN, Grant RW, Adams SH, et al. Endocrine and metabolic effects of consuming fructose- and glucose-sweetened beverages with meals in obese men and women: influence of insulin resistance on plasma triglyceride responses. J Clin Endocrinol Metab. 2009;94:1562-9.

13. Livesey G, Taylor R. Fructose consumption and consequences for glycation, plasma triacylglycerol, and body weight: meta-analyses and meta-regression models of intervention studies. Am J Clin Nutr. 2008;88:1419-37.

14. Berkey CS, Rockett HR, Field AE, Gillman MW, Colditz GA. Sugar-added beverages and adolescent weight change. Obes Res. 2004;12:778-88.

15. Barros CM, Lessa RQ, Grechi MP, Mouco TL, Souza MG, Wiernsperger $\mathrm{N}$, et al. Substitution of drinking water by fructose solution induces hyperinsulinemia and hyperglycemia in hamsters. Clinics. 2007;62:32734.

16. Jurgens H, Haass W, Castaneda TR, Schurmann A, Koebnick C, Dombrowski F, et al. Consuming fructose-sweetened beverages increases body adiposity in mice. Obes Res. 2005;13:1146-56.

17. Livesey G. Fructose ingestion: dose-dependent responses in health research. J Nutr. 2009;139:1246S-52S.
18. Swarbrick MM, Stanhope KL, Elliott SS, Graham JL, Krauss RM, Christiansen MP, et al. Consumption of fructose-sweetened beverages for 10 weeks increases postprandial triacylglycerol and apolipoprotein-B concentrations in overweight and obese women. Br J Nutr. 2008;100:947-52.

19. Le KA, Faeh D, Stettler R, Ith M, Kreis R, Vermathen P, et al. A 4-wk high-fructose diet alters lipid metabolism without affecting insulin sensitivity or ectopic lipids in healthy humans. Am J Clin Nutr. 2006;84:1374-9.

20. Le KA, Ith M, Kreis R, Faeh D, Bortolotti M, Tran C, et al. Fructose overconsumption causes dyslipidemia and ectopic lipid deposition in healthy subjects with and without a family history of type 2 diabetes. Am J Clin Nutr. 2009;89:1760-5.

21. Collison KS, Saleh SM, Bakheet RH, Al-Rabiah RK, Inglis AL, Makhoul NJ, et al. Diabetes of the liver: the link between nonalcoholic fatty liver disease and HFCS-55. Obesity (Silver Spring). 2009;17:2003-13.

22. de Moura RF, Ribeiro C, de Oliveira JA, Stevanato E, de Mello MA. Metabolic syndrome signs in Wistar rats submitted to different highfructose ingestion protocols. Br J Nutr. 2009;101:1178-84.

23. Blakely SR, Hallfrisch J, Reiser S, Prather ES. Long-term effects of moderate fructose feeding on glucose tolerance parameters in rats. $\mathrm{J}$ Nutr. 1981;111:307-14.

24. Kawasaki T, Igarashi K, Koeda T, Sugimoto K, Nakagawa K, Hayashi $\mathrm{S}$, et al. Rats fed fructose-enriched diets have characteristics of nonalcoholic hepatic steatosis. J Nutr. 2009;139:2067-71.

25. Sartorelli DS, Franco LJ, Gimeno SG, Ferreira SR, Cardoso MA. Dietary fructose, fruits, fruit juices and glucose tolerance status in JapaneseBrazilians. Nutr Metab Cardiovasc Dis. 2009;19:77-83.

26. Tsai CJ, Leitzmann MF, Willett WC, Giovannucci EL. Dietary carbohydrates and glycaemic load and the incidence of symptomatic gall stone disease in men. Gut. 2005;54:823-8.

27. Saldana TM, Basso O, Darden R, Sandler DP. Carbonated beverages and chronic kidney disease. Epidemiology. 2007;18:501-6.

28. Krajcovicova-Kudlackova M, Sebekova K, Schinzel R, Klvanova J. Advanced glycation end products and nutrition. Physiol Res. 2002;5:313-6.

29. Sakai M, Oimomi M, Kasuga M. Experimental studies on the role of fructose in the development of diabetic complications. Kobe J Med Sci. 2002;48:125-36

30. Bray GA. Soft drink consumption and obesity: it is all about fructose. Curr Opin Lipidol. 2010;21:51-7.

31. Dills WL, Jr. Protein fructosylation: fructose and the Maillard reaction. Am J Clin Nutr. 1993;58(5 Suppl):779S-87S

32. Hinton DJ, Ames JM. Site specificity of glycation and carboxymethylation of bovine serum albumin by fructose. Amino Acids. 2006;30:425-34.

33. Gul A, Rahman MA, Hasnain SN. Role of fructose concentration on cataractogenesis in senile diabetic and non-diabetic patients. Graefes Arch Clin Exp Ophthalmol. 2009;247:809-14.

34. Miyazawa N, Kawasaki Y, Fujii J, Theingi M, Hoshi A, Hamaoka R, et al. Immunological detection of fructated proteins in vitro and in vivo. Biochem J. 1998;336:101-7. 
35. Tokita Y, Hirayama Y, Sekikawa A, Kotake H, Toyota T, Miyazawa T, et al. Fructose ingestion enhances atherosclerosis and deposition of advanced glycated end-products in cholesterol-fed rabbits. J Atheroscler Thromb. 2005;12:260-7.

36. Brown CM, Dulloo AG, Yepuri G, Montani JP. Fructose ingestion acutely elevates blood pressure in healthy young humans. Am J Physiol Regul Integr Comp Physiol. 2008;294:R730-7.

37. Wang X, Jia X, Chang T, Desai K, Wu L. Attenuation of hypertension development by scavenging methylglyoxal in fructose-treated rats. J Hypertens. 2008;26:765-72.

38. Khosla UM, Zharikov S, Finch JL, Nakagawa T, Roncal C, Mu W, et al. Hyperuricemia induces endothelial dysfunction. Kidney Int. 2005;67:1739-42.

39. Terzuoli L, Porcelli B, Ponticelli F, Marinello E. Purine nucleotide catabolism in rat liver. Certain preliminary aspects of uricase reaction. Nucleosides Nucleotides Nucleic Acids. 2009;28:193-203.

40. Nakagawa T, Hu H, Zharikov S, Tuttle KR, Short RA, Glushakova O, et al. A causal role for uric acid in fructose-induced metabolic syndrome. Am J Physiol Renal Physiol. 2006;290:F625-31.

41. Wiernsperger N, Nivoit P, De Aguiar LG, Bouskela E. Microcirculation and the metabolic syndrome. Microcirculation. 2007;14:403-38.

42. Alzamendi A, Giovambattista A, Raschia A, Madrid V, Gaillard RC, Rebolledo O, et al. Fructose-rich diet-induced abdominal adipose tissue endocrine dysfunction in normal male rats. Endocrine. 2009;35:227-32.

43. Scelles V, Alessi MC, Raccah D, Juhan-Vague I, Vague P. Plasma plasminogen activator inhibitor activity in rats with nutritionally induced insulin resistance. Thromb Haemost. 1995;74:806-7.

44. Kosugi T, Nakamura M, Sunagawa M. Transition of pathophysiological significance of plasminogen activator inhibitor-From a chief player in antiinflammation, antifibrinolysis to that in the development of insulin resistance. Pathophysiology.;17:109-18.

45. Basciano H, Federico L, Adeli K. Fructose, insulin resistance, and metabolic dyslipidemia. Nutr Metab (Lond). 2005;2:5.

46. Hsieh J, Hayashi AA, Webb J, Adeli K. Postprandial dyslipidemia in insulin resistance: mechanisms and role of intestinal insulin sensitivity. Atheroscler Suppl. 2008;9:7-13.

47. Chong MF, Fielding BA, Frayn KN. Mechanisms for the acute effect of fructose on postprandial lipemia. Am J Clin Nutr. 2007;85:1511-20.

48. Mayes PA. Intermediary metabolism of fructose. Am J Clin Nutr. 1993;58(5 Suppl):754S-65S.

49. Topping DL, Mayes PA. The immediate effects of insulin and fructose on the metabolism of the perfused liver. Changes in lipoprotein secretion, fatty acid oxidation and esterification, lipogenesis and carbohydrate metabolism. Biochem J. 1972;126:295-311.

50. Koo HY, Wallig MA, Chung BH, Nara TY, Cho BH, Nakamura MT. Dietary fructose induces a wide range of genes with distinct shift in carbohydrate and lipid metabolism in fed and fasted rat liver. Biochim Biophys Acta. 2008;1782:341-8.

51. Ouyang X, Cirillo P, Sautin Y, McCall S, Bruchette JL, Diehl AM, et al. Fructose consumption as a risk factor for non-alcoholic fatty liver disease. J Hepatol. 2008;48:993-9.

52. Tobey TA, Mondon CE, Zavaroni I, Reaven GM. Mechanism of insulin resistance in fructose-fed rats. Metabolism. 1982;31:608-12.
53. Abid A, Taha O, Nseir W, Farah R, Grosovski M, Assy N. Soft drink consumption is associated with fatty liver disease independent of metabolic syndrome. J Hepatol. 2009;51:918-24

54. Figlewicz DP, Ioannou G, Bennett Jay J, Kittleson S, Savard C, Roth CL. Effect of moderate intake of sweeteners on metabolic health in the rat. Physiol Behav. 2009;98:618-24.

55. Rapin JR, WiernspergerN.Possible links betweenintestinal permeability and food processing: a therapeutic niche for glutamine? Clinics. 2010;in press.

57. Spruss A, Kanuri G, Wagnerberger S, Haub S, Bischoff SC, Bergheim I. Toll-like receptor 4 is involved in the development of fructose-induced hepatic steatosis in mice. Hepatology. 2009;50:1094-104.

58. Skoog SM, Bharucha AE, Zinsmeister AR. Comparison of breath testing with fructose and high fructose corn syrups in health and IBS. Neurogastroenterol Motil. 2008;20:505-11.

59. Corlew-Roath M, Di Palma JA. Clinical impact of identifying lactose maldigestion or fructose malabsorption in irritable bowel syndrome or other conditions. South Med J. 2009;102:1010-2.

60. Choi YK, Kraft N, Zimmerman B, Jackson M, Rao SS. Fructose intolerance in IBS and utility of fructose-restricted diet. J Clin Gastroenterol. 2008;42:233-8.

61. Beyer PL, Caviar EM, McCallum RW. Fructose intake at current levels in the United States may cause gastrointestinal distress in normal adults. J Am Diet Assoc. 2005;105:1559-66.

62. Gibson PR, Newnham E, Barrett JS, Shepherd SJ, Muir JG. Review article: fructose malabsorption and the bigger picture. Aliment Pharmacol Ther. 2007 15;25:349-63.

63. Fernandez-Banares F, Esteve M, Viver JM. Fructose-sorbitol malabsorption. Curr Gastroenterol Rep. 2009;11:368-74.

64. DiMeglio DP, Mattes RD. Liquid versus solid carbohydrate: effects on food intake and body weight. Int $\mathrm{J}$ Obes Relat Metab Disord. 2000;24:794-800

65. Van Wymelbeke V, Beridot-Therond ME, de La Gueronniere V, Fantino $\mathrm{M}$. Influence of repeated consumption of beverages containing sucrose or intense sweeteners on food intake. Eur J Clin Nutr. 2004;58:154-61.

66. DellaValle DM, Roe LS, Rolls BJ. Does the consumption of caloric and non-caloric beverages with a meal affect energy intake? Appetite. 2005;44:187-93.

67. Bantle JP. Dietary fructose and metabolic syndrome and diabetes. J Nutr. 2009;139:1263S-8S.

68. Wiernsperger NF. Oxidative stress: the special case of diabetes. Biofactors. 2003;19:11-8.

69. Sanchez-Lozada LG, Mu W, Roncal C, Sautin YY, Abdelmalek M, Reungjui $\mathrm{S}$, et al. Comparison of free fructose and glucose to sucrose in the ability to cause fatty liver. Eur J Nutr. 2010;49:1-9.

70. Vukovic J, Modun D, Budimir D, Sutlovic D, Salamunic I, Zaja I, et al. Acute, food-induced moderate elevation of plasma uric acid protects against hyperoxia-induced oxidative stress and increase in arterial stiffness in healthy humans. Atherosclerosis. 2009;207:255-60.

71. Bo S, Gambino R, Durazzo M, Ghione F, Musso G, Gentile L, et al. Associations between serum uric acid and adipokines, markers of inflammation, and endothelial dysfunction. J Endocrinol Invest. 2008;31:499-504 
72. Nieto FJ, Iribarren C, Gross MD, Comstock GW, Cutler RG. Uric acid and serum antioxidant capacity: a reaction to atherosclerosis? Atherosclerosis. 2000;148:131-9.

73. Sautin YY, Nakagawa T, Zharikov S, Johnson RJ. Adverse effects of the classic antioxidant uric acid in adipocytes: NADPH oxidase-mediated oxidative/nitrosative stress. Am J Physiol Cell Physiol. 2007;293:C584-96.

74. Yoo HG, Lee SI, Chae HJ, Park SJ, Lee YC, Yoo WH. Prevalence of insulin resistance and metabolic syndrome in patients with gouty arthritis. Rheumatol Int. 2009; 20.

75. Croymans DM, Sanchez A, Barth JD, Roberts CK. Carotid intima-media thickness, dietary intake, and cardiovascular phenotypes in adolescents: relation to metabolic syndrome. Metabolism. 2010;59:533-9.

76. Kramer CK, von Muhlen D, Jassal SK, Barrett-Connor E. Serum uric acid levels improve prediction of incident type 2 diabetes in individuals with impaired fasting glucose: the Rancho Bernardo Study. Diabetes Care. 2009;32:1272-3.

77. Choi HK, Curhan G. Soft drinks, fructose consumption, and the risk of gout in men: prospective cohort study. Bmj. 2008;336:309-12.

78. Lee K. Relationship between uric acid and hepatic steatosis among Koreans. Diabetes Metab. 2009;35:447-51

79. Lin KP. The relationship between serum uric acid concentration and metabolic syndrome in university freshmen. J Nurs Res. 2009;17:286-92.

80. Nakagawa T, Tuttle KR, Short RA, Johnson RJ. Hypothesis: fructoseinduced hyperuricemia as a causal mechanism for the epidemic of the metabolic syndrome. Nat Clin Pract Nephrol. 2005;1:80-6.

81. Chang CH, Chen YM, Chuang YW, Liao SC, Lin CS, Tang YJ, et al Relationship between hyperuricemia (HUC) and metabolic syndrome (MS) in institutionalized elderly men. Arch Gerontol Geriatr. 2009;49 Suppl 2:S46-9.

82. Feig DI, Kang DH, Johnson RJ. Uric acid and cardiovascular risk. N Engl J Med. 2008;359:1811-21.

83. Forman JP, Choi H, Curhan GC. Uric acid and insulin sensitivity and risk of incident hypertension. Arch Intern Med. 2009 26;169:155-62.

84. Kanellis J, Kang DH. Uric acid as a mediator of endothelial dysfunction, inflammation, and vascular disease. Semin Nephrol. 2005;25:39-42.

85. Sundstrom J, Sullivan L, D’Agostino RB, Levy D, Kannel WB, Vasan RS. Relations of serum uric acid to longitudinal blood pressure tracking and hypertension incidence. Hypertension. 2005;45:28-33.

86. Wexler BC, Greenberg BP. Effect of increased serum urate levels on virgin rats with no arteriosclerosis versus breeder rats with preexistent arteriosclerosis. Metabolism. 1977;26:1309-20.

88. Strazzullo P, Puig JG. Uric acid and oxidative stress: relative impact on cardiovascular risk? Nutr Metab Cardiovasc Dis. 2007;17:409-14.

89. Kang DH, Park SK, Lee IK, Johnson RJ. Uric acid-induced C-reactive protein expression: implication on cell proliferation and nitric oxide production of human vascular cells. J Am Soc Nephrol. 2005;16:3553-62.

90. Angelopoulos TJ, Lowndes J, Zukley L, Melanson KJ, Nguyen V, Huffman A, et al. The effect of high-fructose corn syrup consumption on triglycerides and uric acid. J Nutr. 2009;139:1242S-5S.

91. Choi JW, Ford ES, Gao X, Choi HK. Sugar-sweetened soft drinks, diet soft drinks, and serum uric acid level: the Third National Health and Nutrition Examination Survey. Arthritis Rheum. 2008;59:109-16.
93. Sun SZ, Flickinger BD, Williamson-Hughes PS, Empie MW. Lack of association between dietary fructose and hyperuricemia risk in adults. Nutr Metab. (Lond). 2010;7:16

94. Melanson KJ, Zukley L, Lowndes J, Nguyen V, Angelopoulos TJ, Rippe JM. Effects of high-fructose corn syrup and sucrose consumption on circulating glucose, insulin, leptin, and ghrelin and on appetite in normal-weight women. Nutrition. 2007;23:103-12.

95. Murphy SP. The state of the science on dietary sweeteners containing fructose: summary and issues to be resolved. J Nutr. 2009;139:1269S-70S

96. Schaefer EJ, Gleason JA, Dansinger ML. Dietary fructose and glucose differentially affect lipid and glucose homeostasis. J Nutr. 2009; 139:1257S-62S

97. Thresher JS, Podolin DA, Wei Y, Mazzeo RS, Pagliassotti MJ. Comparison of the effects of sucrose and fructose on insulin action and glucose tolerance. Am J Physiol Regul Integr Comp Physiol. 2000;279:R1334-40

98. Soenen S, Westerterp-Plantenga MS. No differences in satiety or energy intake after high-fructose corn syrup, sucrose, or milk preloads. Am J Clin Nutr. 2007;86:1586-94

99. Stanhope KL, Griffen SC, Bair BR, Swarbrick MM, Keim NL, Havel PJ. Twenty-four-hour endocrine and metabolic profiles following consumption of high-fructose corn syrup-, sucrose-, fructose-, and glucose-sweetened beverages with meals. Am J Clin Nutr. 2008;87:1194-203.

100.Ngo Sock ET, Le KA, Ith M, Kreis R, Boesch C, Tappy L. Effects of a short-term overfeeding with fructose or glucose in healthy young males. Br J Nutr. 2009 24:1-5.

101. Stanhope KL, Havel PJ. Fructose consumption: considerations for future research on its effects on adipose distribution, lipid metabolism, and insulin sensitivity in humans. J Nutr. 2009;139:1236S-41S.

102.Anurag P, Anuradha CV. Metformin improves lipid metabolism and attenuates lipid peroxidation in high fructose-fed rats. Diabetes Obes Metab. 2002;4:36-42.

103.Baret G, Peyronnet J, Grassi-Kassisse D, Dalmaz Y, Wiernsperger N, Geloen A. Increased intraabdominal adipose tissue mass in fructose fed rats: correction by metformin. Exp Clin Endocrinol Diabetes. 2002;110:298-303.

104.Faure P, Rossini E, Wiernsperger N, Richard MJ, Favier A, Halimi S. An insulin sensitizer improves the free radical defense system potential and insulin sensitivity in high fructose-fed rats. Diabetes. 1999;48:353-7.

105.Srividhya S, Anuradha CV. Metformin improves liver antioxidant potential in rats fed a high-fructose diet. Asia Pac J Clin Nutr. 2002;1:319-22.

106. Tran LT, Yuen VG, McNeill JH. The fructose-fed rat: a review on the mechanisms of fructose-induced insulin resistance and hypertension. Mol Cell Biochem. 2009;332:145-59.

107.Oda E, Kawai R, Sukumaran V, Watanabe K. Uric acid is positively associated with metabolic syndrome but negatively associated with diabetes in Japanese men. Intern Med. 2009;48:1785-91.

108.Hu FB, Malik VS. Sugar-sweetened beverages and risk of obesity and type 2 diabetes: Epidemiologic evidence. Physiol Behav. 2010;100:4754 